\title{
Prognostic implications of circulating Epstein-Barr virus DNA for extranodal natural killer/T-cell lymphoma, nasal type: a meta-analysis
}

This article was published in the following Dove Press journal: Cancer Management and Research

\section{Ruiwan Chen $1, *$ \\ Chengtao Wangl,* \\ Yu Zhou ${ }^{2, *}$ \\ Bixiu Wen'}

'Department of Radiation Oncology, The First Affiliated Hospital, Sun Yatsen University, Guangzhou 510080 , China; ${ }^{2}$ Department of General Surgery, Guangdong General Hospital, Guangdong Academy of Medical Sciences, Guangzhou 510080, China

*These authors contributed equally to this work
Correspondence: Bixiu Wen Department of Radiation Oncology, The First Affiliated Hospital, Sun Yat-sen University, No. 58, Zhong Shan Er Lu, Guangzhou 510080 , China

Tel +86 2087755766 (ext 8425)

Fax +8687338425

Email wenbix@mail.sysu.edu.cn
Introduction: To evaluate the prognostic value of circulating Epstein-Barr virus DNA for extranodal natural killer/T-Cell lymphoma, nasal type (ENKTL), we performed a meta-analysis of published studies that provided survival information with pre-/post-treatment circulating EBV DNA. Methods: Eligible studies that discussed prognostic significance of circulating EBV DNA in ENKTL were included. Random effects models were applied to obtain the estimated hazard ratios and 95\% confidence intervals to evaluate prognostic significance (OS and DFS/PFS). Eleven studies covering a total of 562 subjects were included in this analysis.

Results: The summary HRs and 95\% CIs of pre-treatment EBV DNA for OS and PFS/DFS were 4.43 (95\% CI 2.66-7.39, $P<0.00001)$ and 3.12 (95\% CI 1.42-6.85, $P=0.005)$, respectively. The corresponding HRs and 95\% CIs of post-treatment EBV DNA for OS and PFS/DFS were 6.28 (95\% CI 2.75-14.35, $P<0.0001)$ and 6.57 (95\% CI 2.14-20.16, $P=0.001)$. Subgroup analyses indicated a strong trend of prognostic powers with pre-/post-treatment EBV DNA.

Conclusion: With the present evidence, circulating EBV DNA consistently correlated with poorer prognosis in patients with ENKTL which need further investigation in large-scale clinical studies.

Keywords: circulating EBV DNA, ENKTL, prognosis

\section{Introduction}

Extranodal natural killer (NK)/T-cell lymphoma, nasal type (ENKTL-NT) is an aggressive malignancy of putative NK-cell origin, with a minority deriving from the T-cell lineage, which presents peculiar clinicopathological features, including angioinvasion, prominent necrosis, and close association with Epstein-Barr virus (EBV). ${ }^{1,2}$ It occurs most commonly (80\%) in the nose and upper aerodigestive tract, and less commonly $(20 \%)$ in the non-nasal areas (skin, gastrointestinal tract, testis, salivary gland). It generally pursues an aggressive clinical course and has a poor outcome. ${ }^{3}$ Despite recent application of PET/CT in diagnosis, ${ }^{4}$ concurrent chemoradiotherapy in early-stage patients, ${ }^{5-7}$ and newly developed chemotherapy regimen containing L-asparaginase, ${ }^{8,9}$ it still presents a poor prognosis without an established standard therapy.

Previous studies have identified several prognostic clinicopathological features of ENKTL, including age, primary sites, Ann Arbor stage, ${ }^{10}$ International prognostic index, ${ }^{11}$ and Korea-developed NK/T-cell prognostic index. ${ }^{12}$ However, varying outcomes have been observed in patients with similar prognostic features and undergoing similar therapies, suggesting inherent heterogeneity of tumor and insufficiency of existing prognostication. ${ }^{13}$ Although several biomarkers have been reported as a prognostic 
surrogate, prognostic prediction in ENKTL is still dismal. Therefore, it is of great clinical value to identify novel biomarkers, which could be utilized as effective prognostic predictors or possible therapeutic targets, in order to optimize the treatment of patients with ENKTL.

$\mathrm{EBV}$ is a human herpes virus that has been confirmed to be in close association with several lymphoid and epithelial malignancies. ${ }^{14,15}$ It has been reported that inappropriate expression of EBV latent genes combined with environment and genetic cofactors may result in virus-associated malignancies. ${ }^{16}$ In EBV-associated nasopharyngeal carcinoma (NPC), the putative tumorigenic role has been thoroughly studied and revealed in large-scale studies that circulating EBV DNA is correlated with tumor load and disease prognosis. ${ }^{17-19}$ Circulating EBV DNA has been detected in plasma or serum in lymphomas, including ENKTL, Hodgkin's disease, AIDS-related lymphoma, and diffuse large B-cell lymphoma. Due to the rarity of ENKTL, there is only several small series of studies that reported the prognostic effect of circulating EBV DNA, with various clinical stages, primary locations and treatment regimens.

Hence, we conducted the present meta-analysis to comprehensively explore the potential prognostic impact of circulating EBV DNA in ENKTL.

\section{Materials and methods}

\section{Search strategy}

We searched systematically PubMed, Embase, the Science Citation Index, Cochrane databases, and the Ovid Database for studies discussed prognostic significance of circulating EBV DNA in ENKTL, with no restrictions on language, place of publication, or date of publication (up to June 2017). The main search terms explored were "Epstein-Barr virus", "EBV", "EBV load", "Natural killer/T-cell lymphoma”, "NK/T-cell lymphoma", and "ENKTL".

\section{Eligibility criteria}

To yield potential relevant publications, we screened the titles and abstracts and author information. Full texts were considered for detailed assessment according to the following inclusion criteria: 1) containing patient cases of ENKTL, 2) measuring the titer of circulating EBV DNA in either plasma or whole blood, and 3) investigating the prognostic significance of EBV DNA titers in ENKTL patients with at least one of the outcome measures of interest. Studies excluded from our study were those that: 1) were duplicated publications, and 2) showed no survival data or insufficient data to be extracted.

\section{Data extraction}

Two investigators (CW and YZ) independently evaluated each paper and extracted data, and any discrepancy between the 2 researchers was resolved via discussion, with a third investigator if necessary. The following information was extracted: first author, publication year, type of studies, population characteristics (i.e., country, number of patients), clinicopathological characteristics (i.e., anatomical sites, tumor stage), sampling time (pre-treatment, intra-treatment, or post-treatment), detection methods (reverse transcriptionpolymerase chain reaction [RT-PCR]), EBV DNA positive rate, median EBV DNA copies, end points, and survival data. For 1 study using 2 different primer sets for LMP1 and Bam HI W fragment by RT-PCR, each of the cohorts was considered an independent data set.

\section{Statistical approaches}

Statistical analysis was performed using Review Manager 5.2 (Copenhagen: The Nordic Cochrane Centre; The Cochrane Collaboration, 2012). ${ }^{22}$ The estimated hazard ratio (HR) and associated 95\% CI were used to evaluate prognostic significance (overall survival [OS] and disease-free survival/ progression-free survival [DFS/PFS]). If the HR and its variance were not reported directly in the original study, these values were calculated based on survival data or survival curves using software designed by Tierney. ${ }^{20}$ The randomeffects model was explored to perform the analyses, because this model obtained more conservative results than the fixed-effect model. ${ }^{21}$ Heterogeneity among the studies was tested using the $\chi^{2}$ test and $I^{2}$ statistic. A value of $I^{2}<25 \%$, within $25 \%-50 \%$, or $>50 \%$ was regarded as low, moderate, or significant heterogeneity, respectively. We then evaluated the potential publication bias with funnel plots. The quality of the included studies was assessed with the Newcastle-Ottawa scale (NOS) for cohort studies. ${ }^{22}$

\section{Results}

\section{Baseline characteristics}

The comprehensive literature search was performed till June 2017, yielding a total of 406 studies. Among these studies, 356 publications were identified as non-English publications, duplicates, or laboratory researches. The remaining 50 studies were thoroughly reviewed, of which 11 studies were appropriate for the meta-analysis (Figure 1).

Eleven studies (sample size from 15 to 120) with 562 patients, basically located in East Asia, were published between 2002 and 2016. ${ }^{23-33}$ All the included publications provided data on the correlation between circulating EBV 


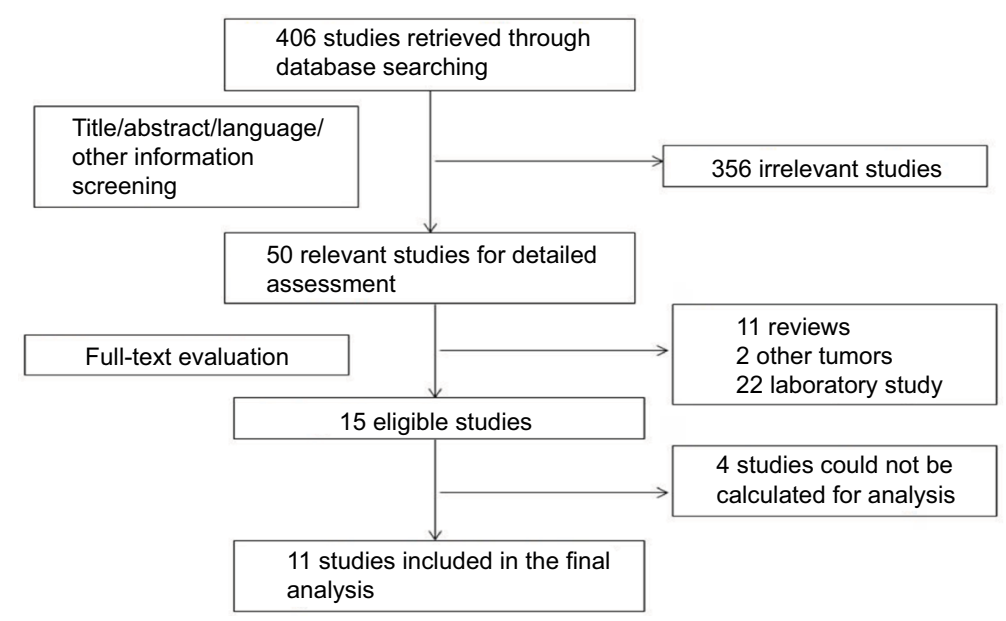

Figure I Selection of studies.

Note: Flow diagram showing the selection process for the enrolled studies.

DNA and prognosis in patients with ENKTL. There were 4 studies designed prospectively ${ }^{25,27,29,30}$ and the remaining retrospectively. The most employed detection methods were RT-PCR, with 1 study from Japan ${ }^{24}$ using 2 different primer sets for LMP1 and Bam HI W fragment, which was considered as 2 independent data sets. For the sample types of included studies, 5 studies used EBV DNA detection in plasma, 1 study used serum, 3 studies from whole blood, 1 study from plasma/MNC, and 1 study from plasma/whole blood. Positive rates of detected EBV DNA irrespective of methods and time points varied from $4.20 \%$ to $100 \%$ in extracted studies. The characteristics of the included studies are summarized in Table 1. The quality of the included studies was evaluated with NOS and summarized in Table 2.

\section{Correlation between EBV DNA and survival outcome Pre-treatment EBV DNA and OS}

A total of 10 cohorts evaluated the relationship between pre-treatment EBV DNA and OS, with 7 cohorts giving the HRs and 95\% CIs for OS directly; the HRs in the remaining studies were extracted using the survival curves with $P$-values. The $\chi^{2}$ test showed low heterogeneity among the studies $\left(P=0.34 ; I^{2}=11 \%\right)$. The combined pooled HR of the aforementioned studies by a random-effects model was 4.43 (95\% CI: 2.66-7.39, $P<0.00001$ ), indicating that high EBV DNA load was significantly associated with a poor OS in patients with ENKTL (Figure 2A).

\section{Pre-treatment EBV DNA and DFS/PFS}

Five articles reporting the correlation between pre-treatment EBV DNA load and DFS/PFS and 6 cohorts were included for meta-analysis. HR and 95\% CI for DFS/PFS were directly extracted from the study reported from 2 cohorts by Ishii et al. The $\chi^{2}$ test showed moderate heterogeneity among the aforementioned studies $\left(P=0.12 ; I^{2}=43 \%\right)$. The pooled HR with a random-effects model was 3.12 (95\% CI: 1.42-6.85, $P=0.005)$, suggesting lower DFS/PFS in ENKTL patients with higher circulating EBV DNA load (Figure 2B).

\section{Post-treatment EBV DNA and OS}

A total of 6 cohorts evaluated the relationship between post-treatment EBV DNA. The $\chi^{2}$ test showed high heterogeneity among the studies $\left(P=0.04 ; I^{2}=57 \%\right)$. The combined pooled HR of the aforementioned studies by a randomeffects model was 6.28 (95\% CI: $2.75-14.35, P<0.0001)$, indicating that high post-treatment EBV DNA load was significantly associated with a poor OS in patients with ENKTL (Figure 3A).

\section{Post-treatment EBV DNA and DFS/PFS}

Four articles reporting the correlation between post-treatment EBV DNA load and DFS/PFS were included for metaanalysis. The $\chi^{2}$ test showed high heterogeneity among the aforementioned studies $\left(P=0.007 ; I^{2}=75 \%\right)$. The pooled HR with a random-effects model was 6.57 (95\% CI: 2.14-20.16, $P=0.001$ ), suggesting a meaningful relationship of a lower DFS/PFS in ENKTL patients with higher post-treatment circulating EBV DNA load (Figure 3B).

\section{Subgroup analysis}

To clarify the intra-study inconsistencies, we further evaluated the relationship between pre-/post-treatment EBV DNA and OS in the following subgroup analysis (Table 3 ). 


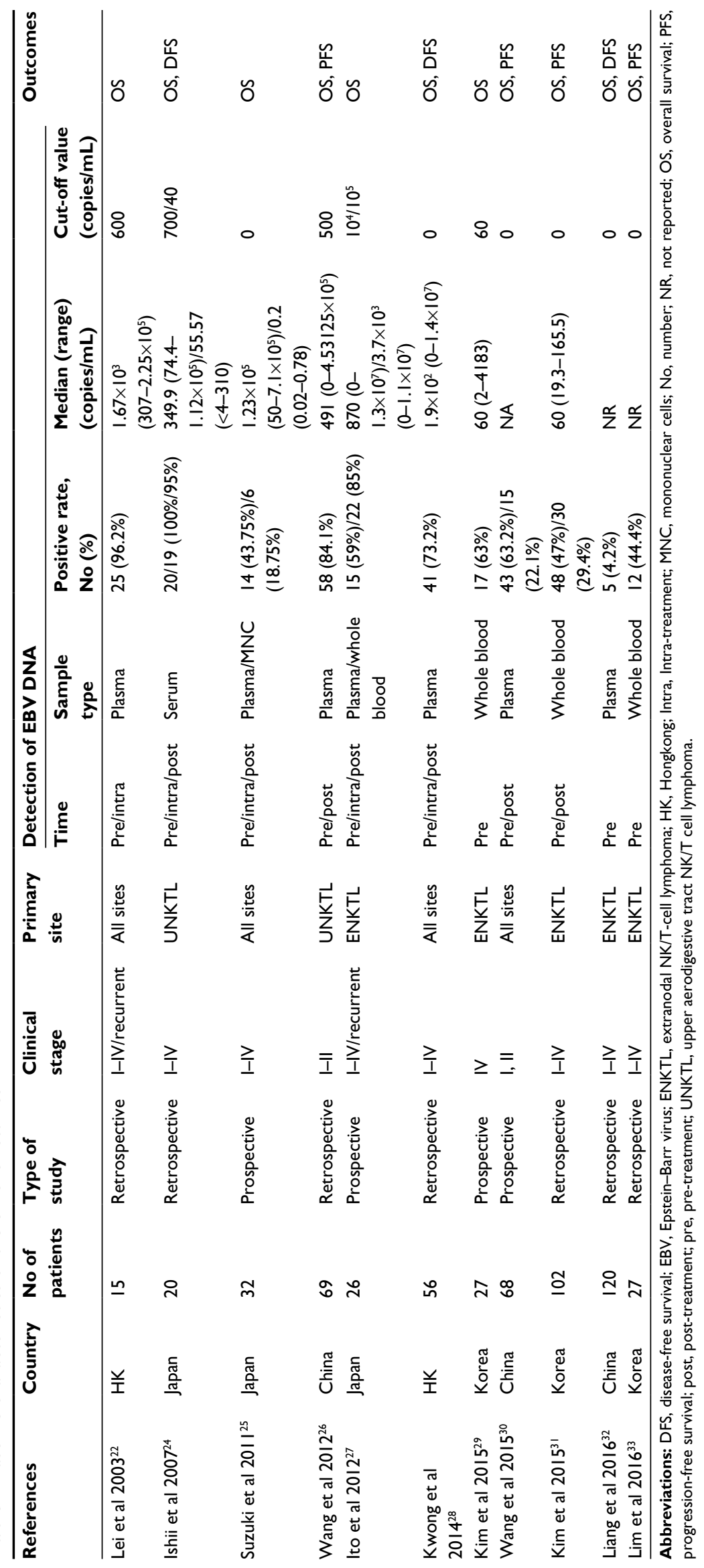


Table 2 Assessment of study quality using the Newcastle-Ottawa scale

\begin{tabular}{|c|c|c|c|c|c|c|c|c|c|c|}
\hline \multirow[t]{2}{*}{ References } & \multicolumn{4}{|c|}{ Selection (0-4) } & \multicolumn{2}{|c|}{ Comparability (0-2) } & \multicolumn{3}{|c|}{ Outcome (0-3) } & \multirow[t]{2}{*}{ Total } \\
\hline & REC & SNEC & AE & DO & $\overline{\text { SC }}$ & AF & $\overline{A O}$ & FU & AFU & \\
\hline Lei et al $2002^{23}$ & I & 1 & I & 1 & 0 & 0 & 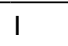 & 0 & 0 & 5 \\
\hline Ishii et al $2007^{24}$ & 0 & I & I & I & 0 & 0 & I & I & I & 6 \\
\hline Suzuki et al $201 I^{25}$ & I & I & I & I & 0 & 0 & I & I & I & 7 \\
\hline Wang et al $2012^{26}$ & 0 & I & I & I & 0 & 0 & I & I & 0 & 5 \\
\hline Ito et al $2012^{27}$ & 0 & I & I & I & 0 & 0 & I & I & 0 & 5 \\
\hline Kwong et al $2014^{28}$ & I & 1 & 1 & I & 0 & 0 & 1 & I & 0 & 6 \\
\hline Kim et al $2015^{29}$ & 0 & I & I & I & 0 & 0 & I & I & I & 6 \\
\hline Wang et al $2015^{30}$ & 0 & I & I & I & 0 & 0 & I & I & I & 6 \\
\hline Kim et al $2015^{31}$ & 0 & 1 & I & I & 0 & 0 & 1 & I & 1 & 6 \\
\hline Liang et al $2016^{32}$ & 0 & I & I & I & 0 & 0 & I & I & I & 6 \\
\hline Lim et al $2016^{33}$ & 0 & I & 1 & I & 0 & 0 & $\mathrm{I}$ & I & I & 6 \\
\hline
\end{tabular}

Notes: "I" means that the study satisfied the item, and "0" means the opposite situation.

Abbreviations: AE, ascertainment of exposure; AF, study controls for any additional factors (chemoradiotherapy, curative resection); AFU, adequacy of follow-up of cohorts; AO, assessment of outcome; DO, demonstration that outcome of interest was not present at start of study; FU, follow-up long enough for outcomes to occur; REC, representativeness of the exposed cohort; SC, study controls for age, sex; SNEC, selection of the non-exposed cohort.

A

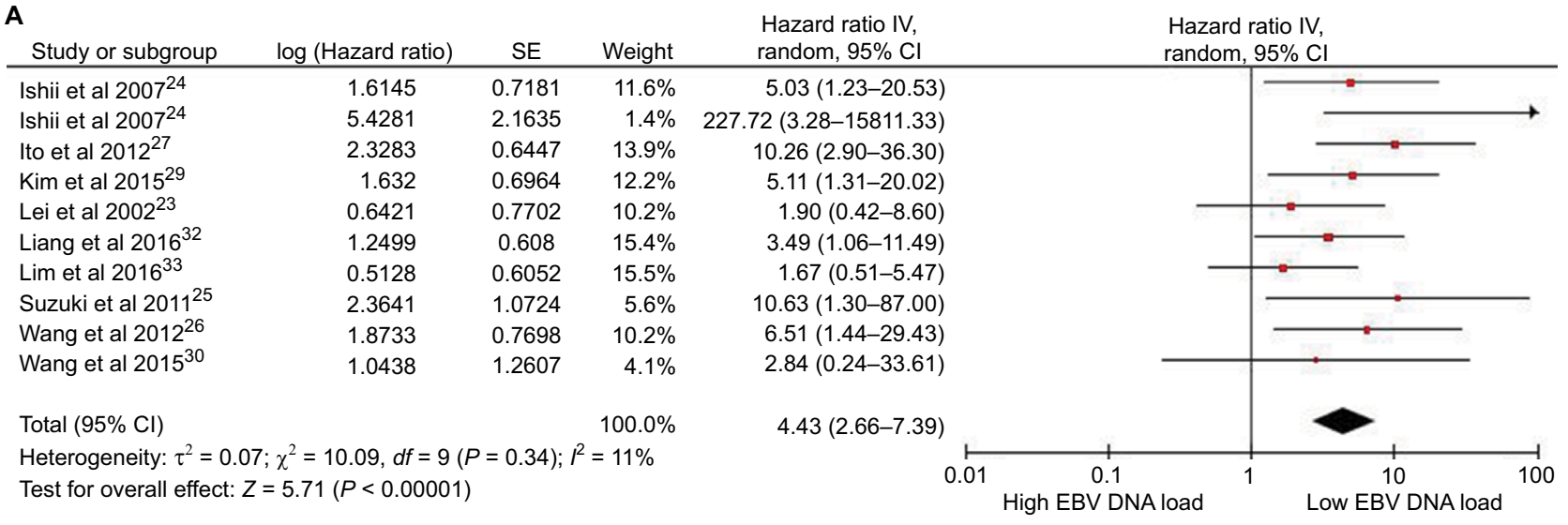

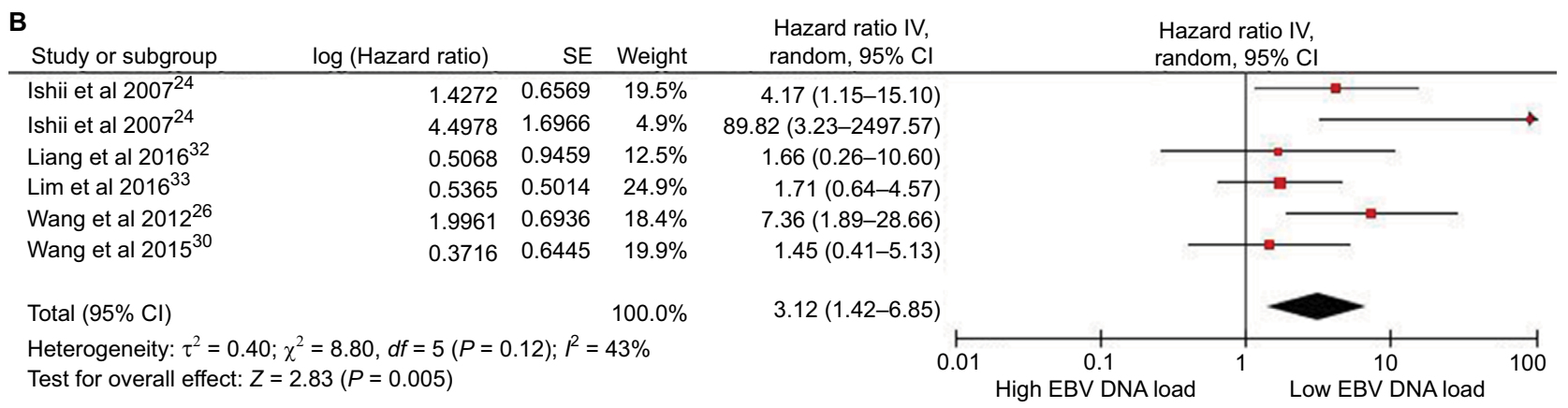

Figure 2 Estimated hazard ratios for OS and PFS/DFS in pre-treatment group.

Notes: (A) Forest plot of OS in pre-treatment group. (B) Forest plot of PFS/DFS in pre-treatment group.

Abbreviations: DFS, disease-free survival; EBV, Epstein-Barr virus; OS, overall survival; PFS, progression-free survival.

\section{Type of samples: plasma/serum and whole blood/mononuclear cell (MNC)}

Pre-treatment EBV DNA is significantly correlated with OS in both plasma/serum and whole blood/MNC subgroups. The HR and 95\% CI for OS in plasma/serum and whole blood/ MNC subgroups were 5.30 (95\% CI: 3.01-9.33, $P<0.00001)$ and 5.30 (95\% CI: $1.68-16.75, P=0.005)$, respectively. The same relationship was found between post-treatment EBV DNA and OS in subgroup analysis.

\section{Tumor sites: upper aerodigestive tract NK/T-cell lymphoma (UNKTL) and others}

When considering primary tumor sites, we further evaluated combined HR and $95 \%$ CI for OS in separated subgroups. 
A

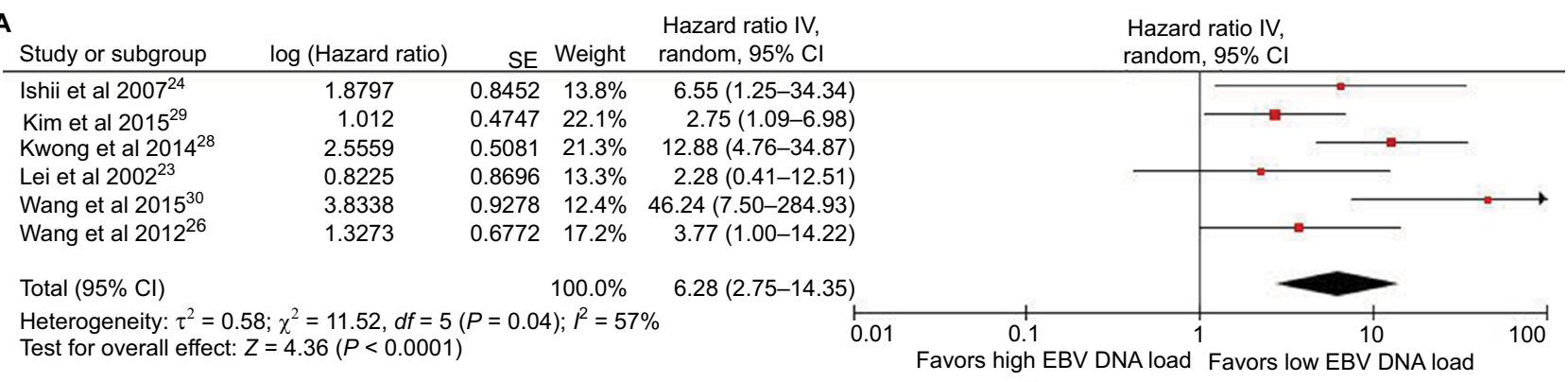

B

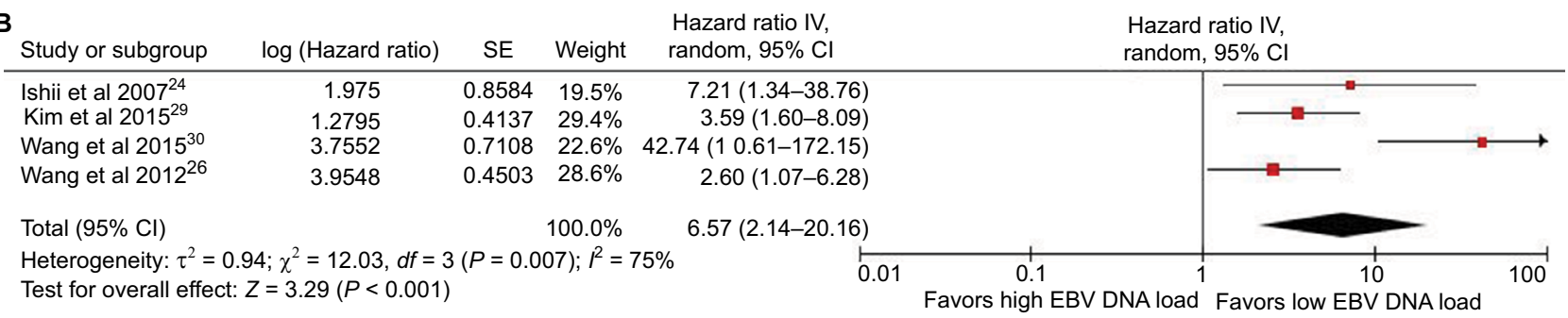

Figure 3 Estimated hazard ratios for OS and PFS/DFS in post-treatment group.

Notes: (A) Forest plot of OS in post-treatment group. (B) Forest plot of PFS/DFS in post-treatment group.

Abbreviations: DFS, disease-free survival; EBV, Epstein-Barr virus; OS, overall survival; PFS, progression-free survival.

Table 3 Results of subgroup analysis on OS

\begin{tabular}{|c|c|c|c|c|c|c|c|c|}
\hline \multirow[t]{2}{*}{ Variables } & \multicolumn{4}{|l|}{ Pre-treatment } & \multicolumn{4}{|l|}{ Post-treatment } \\
\hline & HR (95\% Cl) & $\mathbf{n}$ & $I^{2}(\%)$ & $P$-value & HR (95\% Cl) & $\mathbf{n}$ & $I^{2}(\%)$ & $P$-value \\
\hline \multicolumn{9}{|l|}{ Sample types } \\
\hline Plasma/serum & $5.30(3.01-9.33)$ & 8 & 1 & 0.42 & $8.25(4.43-15.36)$ & 5 & 49 & 0.10 \\
\hline Whole blood/MNC & $5.30(1.68-16.75)$ & 4 & 58 & 0.07 & $2.75(1.09-6.98)$ & I & - & - \\
\hline \multicolumn{9}{|l|}{ Tumor sites } \\
\hline UNKTL & $7.24(1.24-42.10)$ & 3 & 38 & 0.20 & $4.68(1.66-13.19)$ & 2 & 0 & 0.61 \\
\hline Others & $4.15(2.40-7.17)$ & 3 & 8 & 0.37 & $7.29(2.13-24.91)$ & 4 & 73 & 0.01 \\
\hline \multicolumn{9}{|l|}{ Type of study } \\
\hline Prospective & $3.05(1.52-6.11)$ & 6 & 15 & 0.32 & $46.24(7.50-284.93)$ & I & - & - \\
\hline Retrospective & $7.53(3.60-15.78)$ & 4 & 0 & 0.88 & $4.90(2.86-8.40)$ & 5 & 35 & 0.19 \\
\hline \multicolumn{9}{|l|}{ Sample sizes } \\
\hline$N>30$ & $4.81(2.15-10.79)$ & 4 & 0 & 0.76 & $7.75(2.54-23.61)$ & 4 & 71 & 0.02 \\
\hline $\mathrm{N}<30$ & $4.43(1.96-10.06)$ & 6 & 44 & 0.12 & $3.92(1.20-12.86)$ & 2 & 0 & 0.38 \\
\hline
\end{tabular}

Note: “-” = no results.

Abbreviations: HR, hazard ratio; MNC, mononuclear cells; OS, overall survival; UNKTL, upper aerodigestive tract NK/T cell lymphoma.

In UNKTL subgroup, the HR and 95\% CI for OS was 7.24 (95\% CI: $1.24-42.10, P=0.03)$. Meanwhile, in the subgroup in which the primary tumor was not limited to UNKTL, the HR and $95 \% \mathrm{CI}$ for OS was 4.15 (95\% CI: 2.40-7.17, $P<0.00001)$. In included studies evaluating the relationship between posttreatment EBV DNA and OS, the HR and 95\% CI for OS was 4.68 (95\% CI: 1.66-13.19, $P=0.003)$ in ENKTL subgroup, and 7.29 (95\% CI: 2.13-24.91, $P=0.002)$ in the subgroup in which the primary tumor was not limited to ENKTL.

\section{Type of study: prospective and retrospective}

Prospective and retrospective cohorts were both enrolled in this meta-analysis. In further subgroup analysis, the relationship between EBV DNA and OS remains statistically significant in either prospective or retrospective subgroups. The HR and $95 \%$ CI for OS in prospective and retrospective subgroups were 7.53 (95\% CI: $3.60-15.78, P<0.00001)$ and 3.05 (95\% CI: $1.52-6.11, P=0.002)$, respectively. Similar result was found between post-treatment EBV DNA and OS in subgroup analysis.

\section{Sample sizes: $\mathrm{N}>30$ and $\mathrm{N}<30$}

In subgroup with large patient number, the $\mathrm{HR}$ and $95 \% \mathrm{CI}$ for OS was 4.81 (95\% CI: 2.15-10.79, $P=0.0001)$. Meanwhile, in the subgroup with patient number $<30$, the HR and 95\% CI for OS was 4.43 (95\% CI: 1.96-10.06, $P=0.0004)$. When considering the relationship between post-treatment 
EBV DNA and OS, the HR and 95\% CI for OS was 7.75 (95\% CI: 2.54-23.61, $P=0.0003$ ) in subgroup with large patient number and 3.92 (95\% CI: $1.20-12.86, P=0.02)$ in subgroup with patient number $<30$.

\section{Sensitivity analysis and publication bias}

There was no obvious publication bias seen in this metaanalysis (Figure 4A and B)

\section{Discussion}

The standard of care for patients with ENKTL remains controversial. Concurrent chemoradiotherapy, chemotherapy regimen containing L-asparaginase, and stem cell transplantation ${ }^{34}$ have been proposed for patients with ENKTL. However, these high-dose, aggressive therapy strategies do not always translate into survival benefits, with the cumulative probability of 5-year survival ranging from $10 \%$ to $55 \% .^{35-37}$ The optimal treatment strategies and prognostic prediction have not been completely defined yet.

To our knowledge, this is the first meta-analysis to assess the prognostic value of circulating EBV DNA load in patients with ENKTL. Previous small-scale $(\mathrm{N}<60)$ studies have showed that high load of circulating EBV DNA is associated with a poorer survival in ENKTL patients. Hence, a quantitative meta-analysis is urgently required for individualized cancer treatment. Our meta-analysis, which

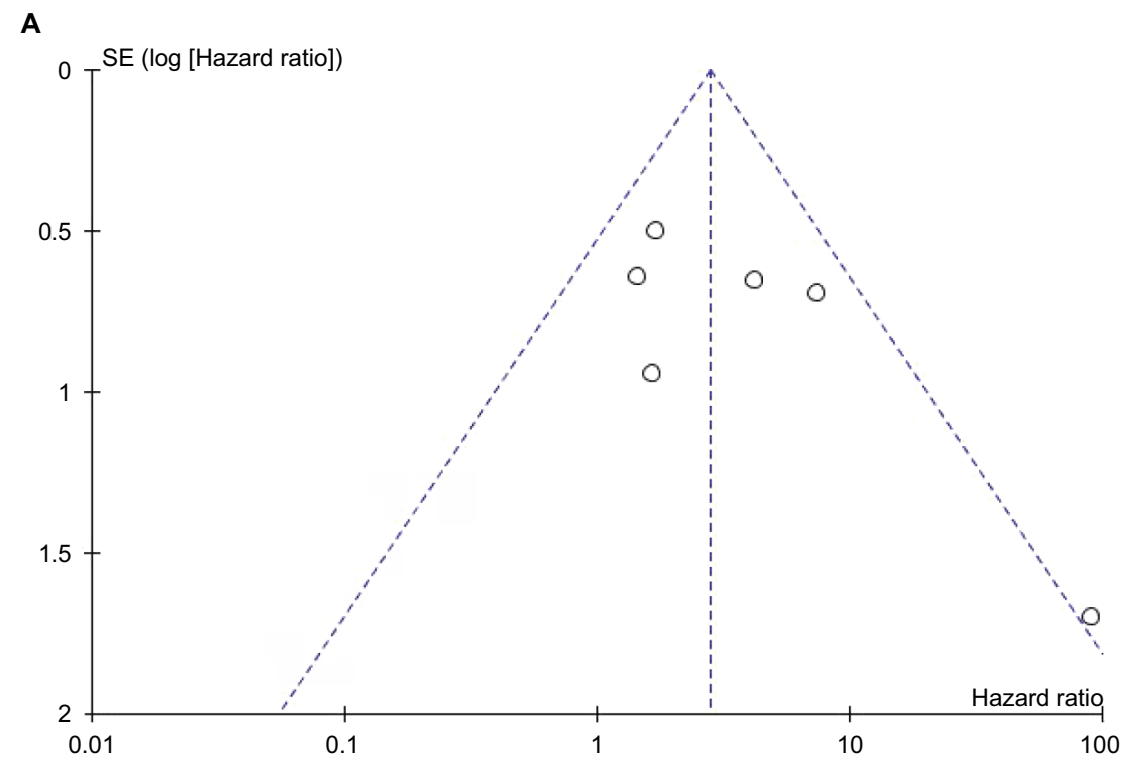

B

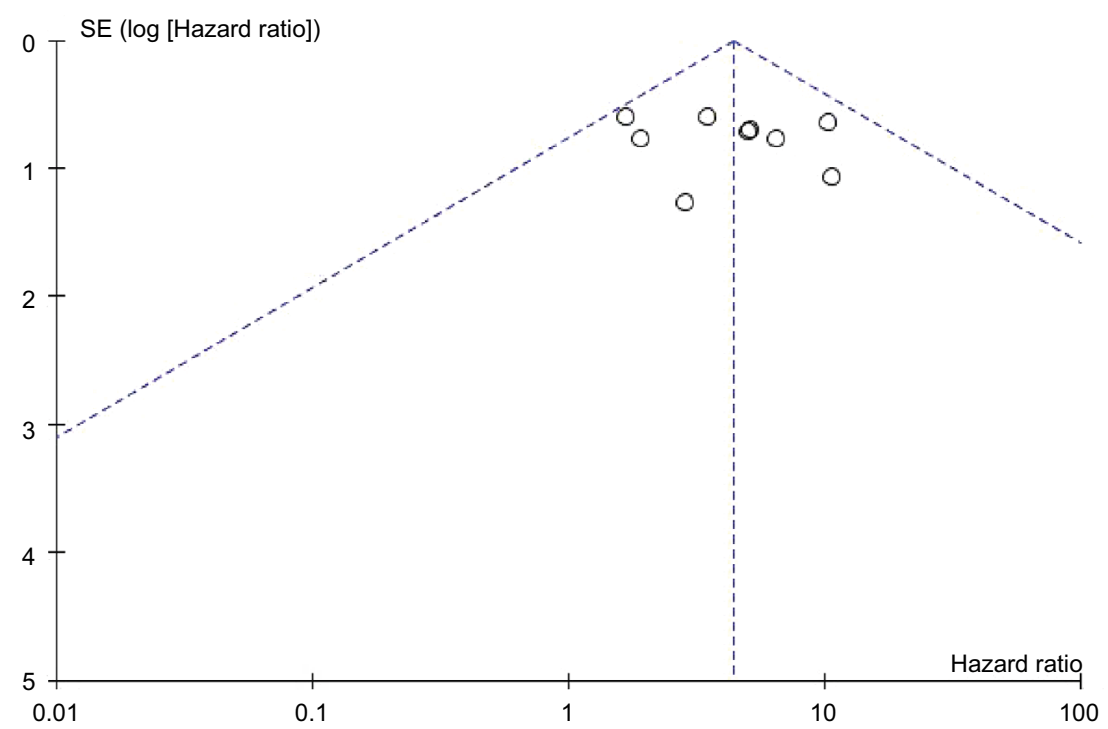

Figure 4 Funnel plots of the enrolled studies.

Notes: (A) OS in pre-treatment group. (B) OS in post-treatment group.

Abbreviation: OS, overall survival. 
involved a relatively large series of patients, provided robust evidence that circulating EBV DNA load in peripheral blood is significantly associated with poor OS and PFS/DFS in patients with ENKTL.

As a well-known EBV-associated malignancy, the invariable association with episomal infection of EBV in ENKTL cells has strongly implied its tumorigenic role. Fragmented viral DNA has also been found in peripheral blood from EBV-associated malignancies, which was mostly $<500$ bp in length. ${ }^{38,39}$ It is reasonable to speculate that measurement of the circulating EBV DNA level may be used as a marker for diagnosis, monitoring treatment response, and prognostication of these EBV-associated malignancies. Previous reports had shown that EBV DNA load has predictive and prognostic significance for EBV-associated malignancies, including NPC ${ }^{40,41}$ and Hodgkin's disease. ${ }^{42,43}$ The diagnostic and prognostic impact has also been evaluated in patients with ENKTL in relatively small-scale studies, suggesting a favorable prognosis with low EBV DNA level. Due to the rarity of the disease, the scales of these cohorts remained relatively small with heterogeneous primary locations, clinical stages, sampling time, and treatment regimens. The present meta-analyses provide stronger evidence that the quantification of EBV DNA level is effective in prognostication in patients with ENKTL.

Besides the potential prognostic impact, other clinical applications of circulating EBV DNA in ENKTL have been evaluated in previous small cohorts of publications, including its diagnostic significance, ${ }^{23,44}$ relationship with clinicopathological features, ${ }^{26}$ and predication of therapy response. ${ }^{27,45}$ Due to the limited data, we did not discuss the aforementioned clinical applications in the present meta-analysis. Large-scale investigation is needed to confirm these potential correlations, which may hypothetically help for patient stratification and personalized therapy.

Circulating EBV DNA can be detected from peripheral plasma, serum, whole blood, or peripheral blood mononuclear cells (PBMNCs). There is still controversy regarding selection of the most suitable blood compartment for the detection of EBV DNA. Previous studies suggested that whole blood compartments and PBMNCs demonstrated a higher sensitivity in diagnosis and prognosis than plasma. ${ }^{46,47}$ However, Spacek et al reported that detection of EBV DNA in plasma was better than in whole blood in predicting prognosis of Hodgkin's disease. ${ }^{48}$ In the present studies, peripheral plasma is mostly used as samples to evaluate EBV DNA level, with 2 cohorts from Korea and Japan using whole blood and another study from Japan using PBMNC. Subgroup analysis shows that regardless of sampling type, circulating EBV DNA level constantly remains strongly correlated with patient prognosis. Comparison of sensitivity and accuracy among each blood compartments is needed for further investigation.

Multiple sampling time points were initially considered in our enrolled studies, including pre-, intra-, and posttreatment. Pre-treatment EBV DNA was mostly applied in majority of included studies, due to the relatively reliable prognostic significance regardless of different therapy regimens. On the other hand, multiple assays of EBV DNA in multiple sample times provided more information, which may be used to analyze the dynamic changes of EBV DNA levels during the treatment. Kwong et al investigated circulating EBV DNA, either baseline or post-treatment, in patients with ENKTL treated with SMILE regimen (comprising steroid, methotrexate, ifosfamide, L-asparaginase, and etoposide) and identified 3 DNA dynamic change patterns. It showed that negative EBV DNA after SMILE with pattern A change in EBV DNA (persistently undetectable) significantly correlated with lower tumor load and superior outcome. ${ }^{28}$ The prognostic impact of EBV DNA in ENKTL is reliable and the considerations for circulating EBV DNA levels before and after treatment remains controversial, which deserves to be investigated in future studies.

It should be pointed out that limitations exist in the metaanalysis that allow us to interpret the results with caution. First, this meta-analysis was based on published data from the enrolled heterogeneous studies, and individual patient data was not available for further investigation. Second, the total number of patients from included studies was relatively small comparing with meta-analysis for other types of cancers. With regard to pediatric patients, 2 cohorts out of 11 studies included patients who were $<14$ years of age with no detailed information on the exact patient number, which might include rare pediatric cases. Therefore, the current meta-analysis included rare pediatric cases that were not enough for further analysis. Considering the rarity of ENKTL worldwide, it is difficult to conduct large prospective studies. High-quality meta-analysis may provide more reliable evidence to guide treatment and predict prognosis. Third, the cut-off value of EBV DNA in our enrolled publications varies from 349.9 copies $/ \mathrm{mL}$ to $6.1 \times 10^{7}$ copies $/ \mathrm{mL}$, implying that there was not a uniform cut-off value to define the high load of circulating EBV DNA in patients with ENKTL, which might lead to heterogeneity.

\section{Conclusion}

In summary, our meta-analysis provides convincing evidence supporting the proposition that circulating EBV 
DNA consistently correlated with prognosis of patients with ENKTL, regardless of primary tumor locations, sampling times, or sample types. However, to confirm this conclusion as well as derived speculations, high-quality, well designed, and large-scale clinical studies of ENKTL are urgently needed.

\section{Novelty and impact statements}

In this work, we have for the first time investigated the prognostic value of circulating EBV DNA for ENKTL by meta-analysis based on published data. Our meta-analysis provides convincing evidence supporting the proposition that circulating EBV DNA consistently correlated with prognosis of patients with ENKTL, regardless of primary tumor locations, sampling times, or sample types. To confirm this conclusion, high-quality, well-designed, and large-scale clinical studies of ENKTL are urgently needed.

\section{Acknowledgments}

This work was supported by National Natural Science Foundation of China (No 81172209 and No 81602048) and Guangdong Natural Science Foundation (No, S2011020003612).

\section{Disclosure}

The authors report no conflicts of interest in this work.

\section{References}

1. Tse E, Kwong YL. How I treat NK/T-cell lymphomas. Blood. 2013;121(25):4997-5005.

2. Zhang T, Fu Q, Gao D, Ge L, Sun L, Zhai Q. EBV associated lymphomas in 2008 WHO classification. Pathol Res Pract. 2014;210(2):69-73.

3. Chan JKC, Quintanilla-Martinez L, Ferry JA, Peh SC. Extranodal NK/T-cell lymphoma, nasal type. In: Swerdlow SH, Campo E, Harris NL, et al, editors. WHO Classification of Tumours of Haematopoietic and Lymphoid Tissues. Lyon, France: IARC; 2008;285-288

4. Zhou X, Lu K, Geng L, Li X, Jiang Y, Wang X. Utility of PET/CT in the diagnosis and staging of extranodal natural killer/T-cell lymphoma: a systematic review and meta-analysis. Medicine (Baltimore). 2014;93(28):e258.

5. Jiang L, Li SJ, Jiang YM, et al. The significance of combining radiotherapy with chemotherapy for early stage extranodal natural killer/Tcell lymphoma, nasal type: a systematic review and meta-analysis. Leuk Lymphoma. 2014;55(5):1038-1048.

6. Kim SJ, Kim K, Kim BS, et al. Phase II trial of concurrent radiation and weekly cisplatin followed by VIPD chemotherapy in newly diagnosed, stage IE to IIE, nasal, extranodal NK/T-cell lymphoma: consortium for improving survival of lymphoma study. J Clin Oncol. 2009;27(35):6027-6032.

7. Yamaguchi M, Tobinai K, Oguchi M, et al. Phase I/II study of concurrent chemoradiotherapy for localized nasal natural killer/T-cell lymphoma: Japan Clinical Oncology Group Study JCOG0211. J Clin Oncol. 2009;27(33):5594-5600.

8. Lin N, Song Y, Zheng W, et al. A prospective phase II study of L-asparaginase-CHOP plus radiation in newly diagnosed extranodal NK/T-cell lymphoma, nasal type. J Hematol Oncol. 2013;6:44.
9. Jiang M, Zhang H, Jiang Y, et al. Phase 2 trial of "sandwich" L-asparaginase, vincristine, and prednisone chemotherapy with radiotherapy in newly diagnosed, stage IE to IIE, nasal type, extranodal natural killer/T-cell lymphoma. Cancer. 2012;118(13):3294-3301.

10. Cheung MM, Chan JK, Lau WH, Ngan RK, Foo WW. Early stage nasal NK/T-cell lymphoma: clinical outcome, prognostic factors, and the effect of treatment modality. Int J Radiat Oncol Biol Phys. 2002;54(1): 182-190.

11. Li YX, Fang H, Liu QF, et al. Clinical features and treatment outcome of nasal-type NK/T-cell lymphoma of Waldeyer ring. Blood. 2008;112(8):3057-3064.

12. Lee J, Suh C, Park YH, et al. Extranodal natural killer T-cell lymphoma, nasal-type: a prognostic model from a retrospective multicenter study. J Clin Oncol. 2006;24(4):612-618.

13. Kim TM, Lee SY, Jeon YK, et al; Lymphoma Subcommittee of the Korean Cancer Study Group. Clinical heterogeneity of extranodal NK/T-cell lymphoma, nasal type: a national survey of the Korean Cancer Study Group. Ann Oncol. 2008;19(8):1477-1484.

14. Lei KI, Chan LY, Chan WY, Johnson PJ, Lo YM. Quantitative analysis of circulating cell-free Epstein-Barr virus (EBV) DNA levels in patients with EBV-associated lymphoid malignancies. Br J Haematol. 2000;111(1):239-246.

15. Leung SF, Zee B, Ma BB, et al. Plasma Epstein-Barr viral deoxyribonucleic acid quantitation complements tumor-node-metastasis staging prognostication in nasopharyngeal carcinoma. J Clin Oncol. 2006;24(34):5414-5418.

16. Williams H, Crawford DH. Epstein-Barr virus: the impact of scientific advances on clinical practice. Blood. 2006;107(3):862-869.

17. Chai SJ,Pua KC, SalehA, etal; Malaysian NPC Study Group. Clinical significance of plasma Epstein-Barr virus DNA loads in a large cohort of Malaysian patients with nasopharyngeal carcinoma. J Clin Virol. 2012;55(1): 34-39.

18. Zhang YX, Kang SY, Chen G, et al. ABO blood group, Epstein-Barr virus infection and prognosis of patients with non-metastatic nasopharyngeal carcinoma. Asian Pac J Cancer Prev. 2014;15(17):7459-7465.

19. Chen M, Yin L, Wu J, et al. Impact of plasma Epstein-Barr virus-DNA and tumor volume on prognosis of locally advanced nasopharyngeal carcinoma. Biomed Res Int. 2015;2015:617949.

20. Tierney JF, Stewart LA, Ghersi D, Burdett S, Sydes MR. Practical methods for incorporating summary time-to-event data into meta-analysis. Trials. 2007;8:16.

21. Schmidt FL, Oh IS, Hayes TL. Fixed- versus random-effects models in meta-analysis: model properties and an empirical comparison of differences in results. Br J Math Stat Psychol. 2009;62(Pt 1): 97-128.

22. Wells GA, Shea B, O'Connell D, et al. The Newcastle-Ottawa Scale (NOS) forassessing the quality if nonrandomized studies inmetaanalyses: Available from: http://www.ohri.ca/programs/clinical_epidemiology/oxford.asp. Accessed August 13, 2014.

23. Lei KI, Chan LY, Chan WY, Johnson PJ, Lo YM. Diagnostic and prognostic implications of circulating cell-free Epstein-Barr virus DNA in natural killer/T-cell lymphoma. Clin Cancer Res. 2002;8(1):29-34.

24. Ishii H, Ogino $\mathrm{T}$, Berger $\mathrm{C}$, et al. Clinical usefulness of serum EBV DNA levels of BamHI W and LMP1 for Nasal NK/T-cell lymphoma. J Med Virol. 2007;79(5):562-72.

25. Suzuki R, Yamaguchi M, Izutsu K, et al; NK-cell Tumor Study Group. Prospective measurement of Epstein-Barr virus-DNA in plasma and peripheral blood mononuclear cells of extranodal NK/T-cell lymphoma, nasal type. Blood. 2011;118(23):6018-6022.

26. Wang ZY, Liu QF, Wang H, et al. Clinical implications of plasma Epstein-Barr virus DNA in early-stage extranodal nasal-type NK/Tcell lymphoma patients receiving primary radiotherapy. Blood. 2012;120(10):2003-2010.

27. Ito Y, Kimura H, Maeda Y, et al. Pretreatment EBV-DNA copy number is predictive of response and toxicities to SMILE chemotherapy for extranodal NK/T-cell lymphoma, nasal type. Clin Cancer Res. 2012;18(15): 4183-4190. 
28. Kwong YL, Pang AW, Leung AY, Chim CS, Tse E. Quantification of circulating Epstein-Barr virus DNA in NK/T-cell lymphoma treated with the SMILE protocol: diagnostic and prognostic significance. Leukemia. 2014;28(4):865-870.

29. Kim SJ, Park S, Kang ES, et al. Induction treatment with SMILE and consolidation with autologous stem cell transplantation for newly diagnosed stage IV extranodal natural killer/T-cell lymphoma patients. Ann Hematol. 2015;94(1):71-78.

30. Wang L, Wang H, Wang JH, et al. Post-treatment plasma EBV-DNA positivity predicts early relapse and poor prognosis for patients with extranodal NK/T cell lymphoma in the era of asparaginase. Oncotarget. 2015;6(30):30317-30326.

31. Kim SJ, Choi JY, Hyun SH, et al; Asia Lymphoma Study Group. Risk stratification on the basis of Deauville score on PET-CT and the presence of Epstein-Barr virus DNA after completion of primary treatment for extranodal natural killer/T-cell lymphoma, nasal type: a multicentre, retrospective analysis. Lancet Haematol. 2015;2(2):e66-e74.

32. Liang R, Wang Z, Bai QX, et al. Natural killer/T cell lymphoma, nasal type: a retrospective clinical analysis in North-Western China. Oncol Res Treat. 2016;39(1-2):45-52.

33. Lim SH, Hyun SH, Kim HS, et al. Prognostic relevance of pretransplant Deauville score on PET-CT and presence of EBV DNA in patients who underwent autologous stem cell transplantation for ENKTL. Bone Marrow Transplant. 2016;51(6):807-812.

34. Ennishi D, Maeda Y, Fujii N, et al. Allogeneic hematopoietic stem cell transplantation for advanced extranodal natural killer/T-cell lymphoma, nasal type. Leuk Lymphoma. 2011;52(7):1255-1261.

35. Suzuki R, Suzumiya J, Yamaguchi M, et al; NK-cell Tumor Study Group. Prognostic factors for mature natural killer (NK) cell neoplasms: aggressive NK cell leukemia and extranodal NK cell lymphoma, nasal type. Ann Oncol. 2010;21(5):1032-1040.

36. Yong W, Zheng W, Zhang Y, et al. L-asparaginase-based regimen in the treatment of refractory midline nasal/nasal-type T/NK-cell lymphoma. Int J Hematol. 2003;78(2):163-167.

37. You JY, Chi KH, Yang MH, et al. Radiation therapy versus chemotherapy as initial treatment for localized nasal natural killer (NK)/T-cell lymphoma: a single institute survey in Taiwan. Ann Oncol. 2004;15(4):618-625.
38. Lo YM, Chan WY, Ng EK, et al. Circulating Epstein-Barr virus DNA in the serum of patients with gastric carcinoma. Clin Cancer Res. 2001;7(7): 1856-1859.

39. Chan KC, Zhang J, Chan AT, et al. Molecular characterization of circulating EBV DNA in the plasma of nasopharyngeal carcinoma and lymphoma patients. Cancer Res. 2003;63(9):2028-2032.

40. Leung SF, Chan KC, Ma BB, et al. Plasma Epstein-Barr viral DNA load at midpoint of radiotherapy course predicts outcome in advanced-stage nasopharyngeal carcinoma. Ann Oncol. 2014;25(6):1204-1208.

41. Lin JC, Wang WY, Chen KY, et al. Quantification of plasma EpsteinBarr virus DNA in patients with advanced nasopharyngeal carcinoma. N Engl J Med. 2004;350(24):2461-2470.

42. Musacchio JG, Carvalho Mda G, Morais JC, et al. Detection of free circulating Epstein-Barr virus DNA in plasma of patients with Hodgkin's disease. Sao Paulo Med J. 2006;124(3):154-157.

43. Kwon JM, Park YH, Kang JH, et al. The effect of Epstein-Barr virus status on clinical outcome in Hodgkin's lymphoma. Ann Hematol. 2006;85(7): 463-468.

44. Au WY, Pang A, Choy C, Chim CS, Kwong YL. Quantification of circulating Epstein-Barr virus (EBV) DNA in the diagnosis and monitoring of natural killer cell and EBV-positive lymphomas in immunocompetent patients. Blood. 2004;104(1):243-249.

45. Kim HS, Kim KH, Chang MH, et al. Whole blood Epstein-Barr virus DNA load as a diagnostic and prognostic surrogate: extranodal natural killer/T-cell lymphoma. Leuk Lymphoma. 2009;50(5):757-763.

46. Stevens SJ, Pronk I, Middeldorp JM. Toward standardization of Epstein-Barr virus DNA load monitoring: unfractionated whole blood as preferred clinical specimen. J Clin Microbiol. 2001;39(4): 1211-1216.

47. Hakim H, Gibson C, Pan J, et al. Comparison of various blood compartments and reporting units for the detection and quantification of Epstein-Barr virus in peripheral blood. J Clin Microbiol. 2007;45(7): 2151-2155.

48. Spacek M, Hubacek P, Markova J, et al. Plasma EBV-DNA monitoring in Epstein-Barr virus-positive Hodgkin lymphoma patients. APMIS. 2011;119(1):10-16.
Cancer Management and Research

\section{Publish your work in this journal}

Cancer Management and Research is an international, peer-reviewed open access journal focusing on cancer research and the optimal use of preventative and integrated treatment interventions to achieve improved outcomes, enhanced survival and quality of life for the cancer patient. The manuscript management system is completely online and includes

\section{Dovepress}

a very quick and fair peer-review system, which is all easy to use. Visit $\mathrm{http}: / / \mathrm{www}$.dovepress.com/testimonials.php to read real quotes from published authors. 